\title{
Potential Trends for COVID-19 Fighting: An Immuno-informatics Overview
}

\author{
Hasanain Abdulhameed Odhar ${ }^{*}$, Salam Waheed Ahjel \\ Department of pharmacy, Al-Zahrawi University College, Karbala, Iraq.
}

*Corresponding author: Hasanain Abdulhameed Odhar, Department of pharmacy, Al-Zahrawi University College, Karbala, Iraq. Tel: 009647725300923, email: hodhar3@gmail.com.

\begin{abstract}
:
Public health care capacity is currently overwhelmed by pandemic outbreak of coronavirus disease 2019. This respiratory disease is a real threat especially for those elderly people with comorbidities. The disease can progress into acute respiratory distress syndrome which can be fatal. No vaccine or drug had been developed against any human coronaviruses due to cessation of previous epidemics and withdrawal of assigned funds. Currently, humanity is in a real challenge to accelerate the development of effective drug and vaccine against severe acute respiratory syndrome coronavirus 2. Till now, computational approaches have played a substantial role for analysis of viral structure and also development of both drug and vaccine candidates in an accelerated pace. Some of these therapeutic designs have found their way into clinical trials. Here, we will overview different immuno-informatics attempts, targets and ideas as possible trends to counteract coronavirus disease 2019 .
\end{abstract}

Keywords: COVID-19, SARS-CoV-2, peptide-based inhibitor, protein-protein interactions, GRP78, epitope, peptide vaccine, antibody variable regions, antibody-dependent enhancement. 


\section{Introduction:}

By the end of 2019, several pneumonia cases of unknown cause were reported in the Chinese city of Wuhan [1]. On January 2020, a novel beta-coronavirus was identified as the etiology pathogen through genomic analysis of throat swab samples collected from some patients [2]. This isolated virus was provisionally known as 2019 novel coronavirus (2019-nCoV), but later was renamed as severe acute respiratory syndrome coronavirus 2 (SARS-CoV-2) [3]. Patients with coronavirus disease 2019 (COVID-19) are usually presented with fever, fatigue, myalgia and dry cough. Some COVID-19 patients may experience dyspnea that can progress into acute respiratory distress syndrome (ARDS) [4]. The transmission of SARS-CoV-2 is mainly through respiratory droplets generated by coughing or sneezing of infected person [5]. COVID19 incubation period can range between 2.1 and 11.1 days with an estimated mean of 6.4 days [6]. The world health organization (WHO) had recognized SARS-CoV-2 outbreak as a global pandemic threat on March 11, 2020 [7]. With no effective drug or vaccine in hands, the reported global confirmed cases of COVID-19 have passed twelve millions on July, 2020 [8].

\section{Review:}

SARS-CoV-2 is a novel beta-coronavirus with a positive sense and single stranded RNA genome. It shares about $79 \%$ of genomic sequence identity with a previously known coronavirus, SARS-CoV [9]. Both viruses use angiotensin converting enzyme 2 (ACE2) as a binding receptor to invade host cells. However, SARS-CoV-2 binds to ACE2 receptor with an estimated affinity greater than SARS-CoV by about 10-20 folds [10]. The role of ACE2 in COVID-19 pathogenesis seems to be implicated beyond viral entry point. It is believed that the pathogenesis of COVID-19 does include ACE2 blockade with subsequent imbalance between angiotensin II and angiotensin-(1-7) levels [11]. No effective drug is currently approved against COVID-19, oxygen-based therapy and management of sepsis are the only available options for ARDS patients. However, clinical trials are currently undertaken to evaluate anti-SARS-CoV2 of several antiviral drugs like Remdesivir [12]. Additionally, virtual modelling and high throughput screening (HTS) have been employed to suggest the repurposing of some FDA approved drugs against SARS-CoV-2 life cycle $[13,14]$. In the same time, no vaccine is available for any coronaviruses as previous development attempts were discontinued due to cessation of SARS-CoV epidemic [15]. In this regard, accelerated efforts are ongoing to generate an effective and safe vaccine against the current pandemic. Different techniques were utilized to produce several SARS-CoV-2 potential vaccine candidates like mRNA based 
vaccine, viral subunit vaccine and viral vector vaccine [16]. From an immuno-informatics perspective, the current review will highlight several attempts, targets and thoughts as potential trends for COVID-19 fighting.

\section{Peptide-based inhibitors of protein-protein interactions:}

Unlike low molecular weight compounds, synthetic peptides have large molecular surface that enable them to interact with the exposed large surface of proteins. These peptides can interfere with the interactions between two protein molecules and thereby influencing a specific biological pathway [17]. This principle of inhibiting protein-protein interactions (PPIs) can be employed to reduce SARS-CoV-2 entry to host cells. The spike protein of SARS-CoV-2 contains the receptor binding domain (RBD) that interacts with angiotensin converting enzyme 2 in host alveolar cell membrane [10]. Recently, two researchers were able to virtually design and evaluate four peptides that may be able to influence the interaction between SARS-CoV-2 spike protein and ACE2 receptor. By computational evaluation of a recently released crystal of SARS-CoV-2 RBD and ACE2 complex [18]; these researchers were capable of recognizing 15 amino acids within SARS-CoV-2 RBD that are essential for the interaction. These specified amino acids residues were used to construct four potential peptide-based inhibitors [19].

We have used Rosetta Peptiderive tool, accessible through ROSIE server, to examine the complex between RBD of SARS-CoV-2 and ACE2 receptor [20,21]. Our preliminary results indicate that Rosetta energy unit (REU) for this proteins pair, expressed as total interface score, is -27.47. Peptiderive tool helped us to identify the peptide fragment of ACE2 receptor that has the largest contribution for interaction energy between ACE2 and spike protein as seen in Figure 1. According to Figure 1 (B), a peptide fragment with a window start position of 4 may contribute to about half (44.46\%) of total interface score (REU). This candidate has a length of 20 amino acids (residues 22-41) and a sequence of "EEQAKTFLDKFNHEAEDLFY". We hope that cyclization with appropriate linker may enhance both efficiency and stability of this linear peptide as a potential peptide-based inhibitor against COVID-19. The red circle in Figure 1 (B) refers to a peptide with a window start position that can be cyclized by disulfide bridge. 
(A)

Spike protein RBD

\section{ACE2}
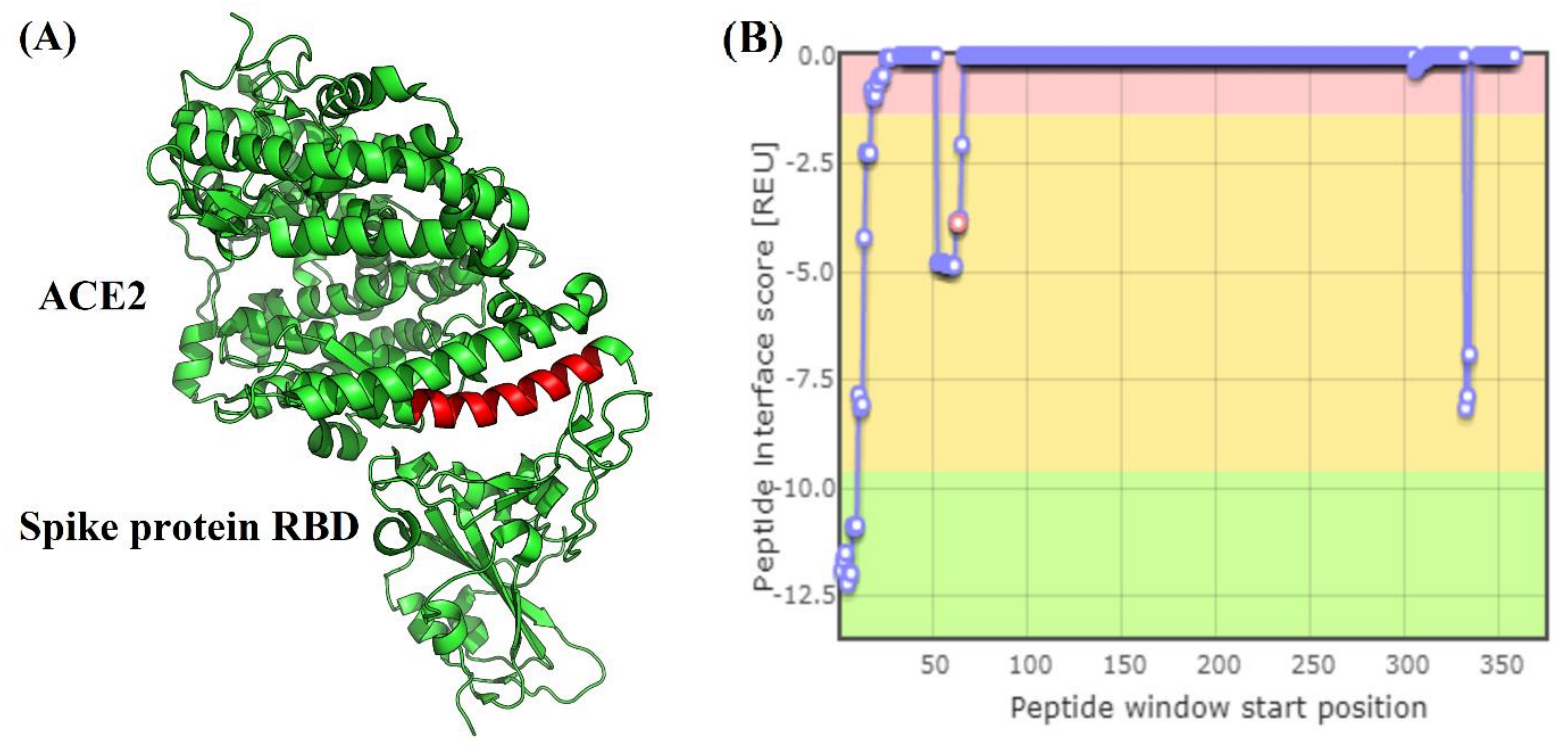

Figure 1: (A) The location of potential peptide-based inhibitor is highlighted in red within $6 \mathrm{M} 0 \mathrm{~J}$ crystal. (B) The energy contribution of the designated peptide can be seen within the plot of interface score versus window start position.

\section{New potential targets for SARS-CoV-2 attachment and entry:}

The tropism of SARS-CoV-2 is mainly dependent on the interaction between viral spike protein and host cellular receptor. SARS-CoV-2 can invade alveolar cells through binding of viral spike protein with angiotensin converting enzyme 2 (ACE2) of host cell. A virtual modelling study proposed that spike protein of SARS-CoV-2 can also bind to glucose regulated protein 78 (GRP78) expressed on host cell membrane [22]. Glucose regulated protein 78 is mainly located within endoplasmic reticulum lumen, it has a chaperone activity. The expression of GRP78 can be induced in response to stressful conditions like cancer and atherosclerosis. This heat shock protein can be also translocated to cell membrane in response to stress where it can play a role in regulating oncogenic pathways [23]. Cell surface GRP78 can act as a co-receptor for Dengue virus and Coxsackievirus A9 [24,25]. The Middle East respiratory syndrome coronavirus (MERS-CoV), another beta-coronavirus, can bind to GRP78 expressed on cell surface. Through interaction with spike protein, GRP78 can augment the entry of MERS-CoV to susceptible cells [26]. Based on the above facts, we believe that patients with chronic diseases are more vulnerable to infection with SARS-CoV-2 in part due to persistent cellular stressful conditions. GRP78 as a stress sensor will be induced and translocated to cellular membrane in those patients, leading to subsequent enhancement of SARS-CoV-2 attachment. 
The role of cell surface GRP78 in SARS-CoV-2 pathogenesis should be further explored, then the rationale to design an inhibitor for this protein-protein interaction can be justified.

In another attempt to disrupt SARS-CoV-2 entry to host cells, a biotech company called BenevolentAI had used machine learning to analyze medical literatures and build a knowledgebased graph that identify new relationships and ways to manipulate disease process. Thus, the team had suggested the use of baricitinib as an inhibitor of receptor mediated endocytosis to reduce SARS-CoV-2 entry. Baricitinib, an antirheumatic drug, can inhibit an enzyme called AP2-associated protein kinase 1 (AAK1) that is mainly involved in receptor mediated endocytosis [27].

\section{Towards epitopes identification and SARS-CoV-2 immune therapy:}

In silico prediction tools of epitopes are keep evolving by using algorithms well trained on experimentally resolved antigen-antibody complexes. For successful recognition by B-cells, these predicted epitopes must be surface exposed with a significant antigenic potential [28]. Recent immuno-informatics attempts have suggested several potential epitopes for B-cells and T-cells against SARS-CoV-2 spike protein [29,30]. Some of these predicted epitopes have enabled virtual designing of either epitope-based peptide vaccine candidates or antibody variable regions. Peptide vaccine design did involve linking predicted epitopes with appropriate peptide linker and addition of suitable adjuvant to improve efficiency $[31,32]$. On the other hand, virtual designing of variable regions for antibody prototype relied on simulating the recombination of VDJ genes by using OptMAVEn-2.0 tool. Then the initial design was further refined by using Rosetta-based in silico affinity maturation to enhance binding affinity. It is believed that these variable regions designs can be later grafted onto a human framework Fc fragment to construct a potential antibody [33].

However, both in vitro and in vivo assessments of these designs seem to be essential as they may elicit the generation of sub-neutralizing or non-neutralizing antibodies inside the body. These antibodies may induce sustained inflammatory reaction or even promote the infectivity of subsequent SARS-CoV-2 infection through antibody-dependent enhancement (ADE) condition. It is believed that amino acid residues with sequence 597-603 within SARS-CoV spike protein can promote ADE condition. This designated sequence is also well conserved in SARS-CoV-2 and therefore it should be eliminated when designing a vaccine or antibody candidates $[16,34]$. 


\section{Conclusion:}

If drugs industry and health organizations didn't cut funding for SARS-CoV vaccine and drug development projects after cessation of epidemic, then may be now we have something in hand to combat or mitigate SARS-CoV-2 pandemic. The efficiency of current procedures of lockdown and social distancing to slow down pandemic dynamics are questionable. The impact of quarantine on global economy and people psychological status must be determined for later resolve. Currently, medicine industry is racing time to release anti-COVID-19 drug or vaccine as soon as possible. But the main question is when we can see a light for ending this gloomy tunnel?

\section{References:}

1. Lu H, Stratton CW, Tang YW. Outbreak of pneumonia of unknown etiology in Wuhan, China: The mystery and the miracle. J Med Virol. 2020 Apr 1;92(4):401-2.

2. Hui DS, I Azhar E, Madani TA, Ntoumi F, Kock R, Dar O, et al. The continuing 2019nCoV epidemic threat of novel coronaviruses to global health - The latest 2019 novel coronavirus outbreak in Wuhan, China. Int J Infect Dis. 2020 Feb 1;91:264-6.

3. Lai CC, Shih TP, Ko WC, Tang HJ, Hsueh PR. Severe acute respiratory syndrome coronavirus 2 (SARS-CoV-2) and coronavirus disease-2019 (COVID-19): The epidemic and the challenges. Int J Antimicrob Agents. 2020 Mar 1;55(3).

4. Guan W, Ni Z, Hu Y, Liang W, Ou C, He J, et al. Clinical Characteristics of Coronavirus Disease 2019 in China. N Engl J Med [Internet]. 2020 Apr 30 [cited 2020 Jun 11];382(18):1708-20. Available from: http://www.nejm.org/doi/10.1056/NEJMoa2002032

5. Han Q, Lin Q, Jin S, You L. Coronavirus 2019-nCoV: A brief perspective from the front line. J Infect. 2020 Apr 1;80(4):373-7.

6. Backer JA, Klinkenberg D, Wallinga J. Incubation period of 2019 novel coronavirus (2019- nCoV) infections among travellers from Wuhan, China, 2028 January 2020. Eurosurveillance. 2020 Feb 6;25(5).

7. WHO Director-General's opening remarks at the media briefing on COVID-19-11 March 2020 [Internet]. [cited 2020 Jun 12]. Available from: https://www.who.int/dg/speeches/detail/who-director-general-s-opening-remarks-atthe-media-briefing-on-covid-19---11-march-2020

8. COVID-19 Map - Johns Hopkins Coronavirus Resource Center [Internet]. [cited 2020 Jul 10]. Available from: https://coronavirus.jhu.edu/map.html

9. Lu R, Zhao X, Li J, Niu P, Yang B, Wu H, et al. Genomic characterisation and epidemiology of 2019 novel coronavirus: implications for virus origins and receptor binding. Lancet. 2020 Feb 22;395(10224):565-74.

10. Wrapp D, Wang N, Corbett KS, Goldsmith JA, Hsieh C-L, Abiona O, et al. Cryo-EM structure of the 2019-nCoV spike in the prefusion conformation. Science (80- ) [Internet]. 2020 Mar 13;367(6483):1260 LP - 1263. Available from: 
http://science.sciencemag.org/content/367/6483/1260.abstract

11. Shete A. Urgent need for evaluating agonists of Angiotensin-(1-7)/ Mas receptor axis for treatment of patients with COVID-19. Int J Infect Dis. 2020 Jul 1;96:348-51.

12. Wang Y, Zhang D, Du G, Du R, Zhao J, Jin Y, et al. Remdesivir in adults with severe COVID-19: a randomised, double-blind, placebo-controlled, multicentre trial. Lancet. 2020 May 16;395(10236):1569-78.

13. Odhar HA, Ahjel SW, Albeer AAMA, Hashim AF, Rayshan AM, Humadi SS.

Molecular docking and dynamics simulation of FDA approved drugs with the main protease from 2019 novel coronavirus. Bioinformation. 2020 Mar 31;16(3):236-44.

14. Yang C-W, Peng T-T, Hsu H-Y, Lee Y-Z, Wu S-H, Lin W-H, et al. Repurposing old drugs as antiviral agents for coronaviruses. Biomed J [Internet]. 2020 May 23 [cited 2020 Jun 13]; Available from:

https://linkinghub.elsevier.com/retrieve/pii/S2319417020300664

15. Harapan H, Itoh N, Yufika A, Winardi W, Keam S, Te H, et al. Coronavirus disease 2019 (COVID-19): A literature review. J Infect Public Health. 2020 May 1;13(5):66773.

16. Shih HI, Wu CJ, Tu YF, Chi CY. Fighting COVID-19: A quick review of diagnoses, therapies, and vaccines. Biomed J. 2020 May 30;

17. Wójcik P, Berlicki Ł. Peptide-based inhibitors of protein-protein interactions. Bioorganic Med Chem Lett. 2016 Feb 1;26(3):707-13.

18. RCSB PDB - 6M17: The 2019-nCoV RBD/ACE2-B0AT1 complex [Internet]. [cited 2020 Jun 14]. Available from: https://www.rcsb.org/structure/6M17

19. Han Y, Král P. Computational Design of ACE2-Based Peptide Inhibitors of SARSCoV-2. ACS Nano. 2020 Apr 28;14(4):5143-7.

20. Sedan Y, Marcu O, Lyskov S, Schueler-Furman O. Peptiderive server: derive peptide inhibitors from protein-protein interactions. Nucleic Acids Res [Internet]. 2016 May 3;44(W1):W536-41. Available from: https://doi.org/10.1093/nar/gkw385

21. RCSB PDB - 6M0J: Crystal structure of SARS-CoV-2 spike receptor-binding domain bound with ACE2 [Internet]. [cited 2020 Jun 14]. Available from: https://www.rcsb.org/structure/6M0J

22. Ibrahim IM, Abdelmalek DH, Elshahat ME, Elfiky AA. COVID-19 spike-host cell receptor GRP78 binding site prediction. J Infect [Internet]. 2020 May 1 [cited 2020 Jun 14];80(5):554-62. Available from: https://linkinghub.elsevier.com/retrieve/pii/S0163445320301079

23. Lee AS. Glucose-regulated proteins in cancer: Molecular mechanisms and therapeutic potential. Nat Rev Cancer. 2014;14(4):263-76.

24. Jindadamrongwech S, Thepparit C, Smith DR. Identification of GRP 78 (BiP) as a liver cell expressed receptor element for dengue virus serotype 2. Arch Virol. 2004 May;149(5):915-27.

25. Triantafilou K, Fradelizi D, Wilson K, Triantafilou M. GRP78, a Coreceptor for Coxsackievirus A9, Interacts with Major Histocompatibility Complex Class I 
Molecules Which Mediate Virus Internalization. J Virol. 2002 Jan 15;76(2):633-43.

26. Chu H, Chan CM, Zhang X, Wang Y, Yuan S, Zhou J, et al. Middle East respiratory syndrome coronavirus and bat coronavirus HKU9 both can utilize GRP78 for attachment onto host cells. J Biol Chem. 2018 Jul 27;293(30):11709-26.

27. Richardson P, Griffin I, Tucker C, Smith D, Oechsle O, Phelan A, et al. Baricitinib as potential treatment for 2019-nCoV acute respiratory disease. Lancet. $2020 \mathrm{Feb}$ 15;395(10223):e30-1.

28. Sanchez-Trincado JL, Gomez-Perosanz M, Reche PA. Fundamentals and Methods for T- and B-Cell Epitope Prediction. Selvan SR, editor. J Immunol Res [Internet]. 2017;2017:2680160. Available from: https://doi.org/10.1155/2017/2680160

29. Odhar HA, Ahjel SW, Humadi SS. Towards the design of epitope candidates for Coronavirus 2. Bioinformation. 2020;16(5):375-86.

30. Joshi A, Joshi BC, Mannan MA ul, Kaushik V. Epitope based vaccine prediction for SARS-COV-2 by deploying immuno-informatics approach. Informatics Med Unlocked. 2020 Jan 1;19:100338.

31. K AP, T S, S K, A VS, Tc V. Design of multi-epitope vaccine candidate against SARS-CoV-2: a In-Silico study. J Biomol Struct Dyn [Internet]. 2020 May 18 [cited 2020 Jun 16];1-10. Available from: http://www.ncbi.nlm.nih.gov/pubmed/32419646

32. Odhar HA, Ahjel SW, Humadi SS. Towards the design of multiepitope-based peptide vaccine candidate against SARS-CoV-2. bioRxiv [Internet]. $2020 \mathrm{Jul} 8$ [cited $2020 \mathrm{Jul}$ 11];2020.07.07.186122. Available from:

http://biorxiv.org/content/early/2020/07/08/2020.07.07.186122.abstract

33. Boorla VS, Chowdhury R, Maranas CD. De novo design of high-affinity antibody variable regions ( $\mathrm{scFv}$ ) against the SARS-CoV-2 spike protein. bioRxiv. 2020 May $11 ; 2020.04 .09 .034868$.

34. Wang Q, Zhang L, Kuwahara K, Li L, Liu Z, Li T, et al. Immunodominant SARS coronavirus epitopes in humans elicited both enhancing and neutralizing effects on infection in non-human primates. ACS Infect Dis. 2016 May 13;2(5):361-76. 